\title{
Image Features Based on a New Approach to 2D Rotation Invariant Quadrature Filters ${ }^{\star}$
}

\author{
Michael Felsberg ${ }^{1}$ and Gerald Sommer ${ }^{2}$ \\ 1 Linköping University, Linköping S-58183, Sweden, \\ mfe@isy.liu.se, www.isy.liu.se/ $\mathrm{mfe}$ \\ 2 Christian-Albrechts-University of Kiel, Kiel D-24105, Germany, \\ gs@ks.informatik. uni-kiel.de, www.ks.informatik. uni-kiel.de/〜gs
}

\begin{abstract}
Quadrature filters are a well known method of low-level computer vision for estimating certain properties of the signal, as there are local amplitude and local phase. However, 2D quadrature filters suffer from being not rotation invariant. Furthermore, they do not allow to detect truly $2 \mathrm{D}$ features as corners and junctions unless they are combined to form the structure tensor. The present paper deals with a new 2D generalization of quadrature filters which is rotation invariant and allows to analyze intrinsically $2 \mathrm{D}$ signals. Hence, the new approach can be considered as the union of properties of quadrature filters and of the structure tensor. The proposed method first estimates the local orientation of the signal which is then used for steering some basis filter responses. Certain linear combination of these filter responses are derived which allow to estimate the local isotropy and two perpendicular phases of the signal. The phase model is based on the assumption of an angular band-limitation in the signal. As an application, a simple and efficient point-of-interest operator is presented and it is compared to the Plessey detector.
\end{abstract}

Keywords: image features, quadrature filters, analytic signal, structure tensor, point-of-interest operator, orientation estimation

\section{Introduction}

This section deals with some basic ideas about quadrature filters and describes related approaches which occur in the literature.

\subsection{Quadrature Filters and Feature Detection}

Quadrature filters are a well known issue in signal processing and low-level computer vision. They are suited for estimating the local amplitude and the local phase of signals. Whereas the local amplitude is a measure for the local intensity of a structure, the local phase describes the structure or shape of the signal [1]. For 1D signals, quadrature filters are obtained by a bandpass filter and its

\footnotetext{
* This work has been developed during M. Felsberg's PhD studies in Kiel, and it has been supported by German National Merit Foundation and by DFG Graduiertenkolleg No. 357 (M. Felsberg) and by DFG Grant So-320-2-2 (G.Sommer).
} 
Hilbert transform which form a pair of an even and an odd filter. This Hilbert pair of filters can be used to detect certain shapes of signals, e.g., peaks and jumps. Furthermore, the local phase allows to distinguish or to classify detected structures. The amplitude response of a quadrature filter is chosen as a bandpass filter in order to isolate certain frequency components, i.e., to reduce the original signal to a signal with small bandwidth, which is necessary to obtain a reasonable interpretation of the local phase [2], page 171. The local amplitude and the local phase can be considered as features of the signal. They are obtained by applying some postprocessing (a change to polar coordinates) to the quadrature filter responses and they locally characterize the signal, at least if they are considered for several scales (for a more detailed discussion on features, see e.g. [3]).

For image processing, it is also desirable to have such an approach which simultaneously allows to detect and to classify image features. Even without going into mathematical details it is obvious that the $2 \mathrm{D}$ generalization of quadrature filters is far from being trivial. Consider for example the classes of $2 \mathrm{D}$ structures which correspond to 1D peaks and jumps. Lines and edges are projections of the former signals but they are themselves intrinsically 1D, i.e., they differ with respect to one direction only [4]. This is different for corners, line-crossings, and general junctions which are all i2D structures. This qualitative extension of structures should be reflected in the applied 2D feature set, such that it should not just contain 2D projections of $1 \mathrm{D}$ features but it should also include a new quality of features which are intrinsically $2 \mathrm{D}$.

Besides this new quality of features, a further degree of freedom for the features is introduced: the orientation of the feature. For the detection and classification of features it is reasonable to have a rotation invariant approach since the orientation information neither affects the intensity nor the classification of a feature. Nevertheless, the orientation information is worth to be reflected in an additional distinct component. Using such an approach instead of a set of orientation dependent operators extends the invariance - equivariance idea [2] to three parts of information about a feature: classification, intensity, and orientation. An appropriate generalization of quadrature filters to $2 \mathrm{D}$ should take into account the previous considerations, at least if the 2D quadrature filters are designed with respect to the application of feature detection and classification.

\section{$1.2 \quad 2 \mathrm{D}$ Quadrature Filters}

Unfortunately, all approaches for 2D quadrature filters ignore one or several of the formerly proposed properties. Except for the quaternionic analytic signal [5], no $2 \mathrm{D}$ extension of the quadrature principle exists which explicitly deals with the intrinsic dimension of the considered signal. However, according to the previous discussion, it is not sufficient just to project $1 \mathrm{D}$ quadrature filters onto $2 \mathrm{D}$ space.

A projection of $1 \mathrm{D}$ quadrature filters to $2 \mathrm{D}$ is performed if the partial Hilbert transform is used to create the odd filter. The partial Hilbert transform is obtained by projecting the frequency vector onto a preference direction and applying the frequency response of the 1D Hilbert transform to this scalar product 
[2]. Special cases of the partial Hilbert transform are the Hilbert transforms with respect to the coordinate axes. The symmetry of the partial Hilbert transform is illustrated in Fig. 1, left. Quadrature filters obtained from the partial Hilbert transform are obviously not rotation invariant and are not adequate for detecting intrinsically $2 \mathrm{D}$ features. It should be mentioned that the steerable quadrature filters proposed in [6] do not have the drawback of being rotation variant since they are orientation adaptive. However, they are not capable to deal with intrinsically $2 \mathrm{D}$ signals either.

A second $2 \mathrm{D}$ extension of quadrature filters is obtained by means of the total Hilbert transform which is just the successive application of the Hilbert transforms with respect to both coordinate axes [7]. The resulting symmetry of the total Hilbert transform is even with respect to point symmetry and odd with respect to line-symmetry (see Fig. 1, second from the left). Quadrature filters obtained from the total Hilbert transform are obviously not rotation invariant and are not capable of detecting intrinsically $1 \mathrm{D}$ features.

A further quadrature approach is obtained by combining the previous two methods. The resulting quadrature filter is only non-zero in the first quadrant of the frequency domain [7]. Unfortunately, the reduction to one quadrant yields a loss of information and therefore, Hahn suggests to consider a second operator output which is non-zero either in the second or in the fourth quadrant. However, the representation in two complex signals is not totally satisfactory and therefore, Bülow and Sommer proposed to use the quaternionic Fourier transform $[8,9]$ instead of the complex one. The resulting quaternionic analytic signal consists of four parts instead of two [5]. Two parts correspond to the partial Hilbert transforms with respect to the coordinate axes and one corresponds to the total Hilbert transform (see Fig. 1, third, fourth, and fifth from the left). The phase approach of the quaternionic analytic signal also reflects the intrinsic dimension to some extent. However, the quaternionic analytic signal is not rotation invariant.

The only non-steered, rotation invariant approach to quadrature filters which occurred in the literature so far, is obtained from the monogenic signal [10]. It is adequate for treating intrinsically $1 \mathrm{D}$ signals but delivers no information about the $2 \mathrm{D}$ part of a signal. The monogenic signal is based on the Riesz transform which is a $2 \mathrm{D}$ generalization of the Hilbert transform. The Riesz transform is antisymmetric with respect to the origin since its frequency response is basically given by the normalized frequency vectors [11] (see also Fig. 1, right). Note that the monogenic signal contains no steering by the orientation but the latter is obtained as an additional feature.

Hence, it is desirable to combine the rotation invariance of the monogenic signal with the symmetry decomposition of the quaternionic analytic signal. This combination directly leads to the new approach which is the main topic of this paper. In order to visualize the drawbacks of the previously mentioned methods, the local amplitudes of the filter responses to the signal $f(x, y)=\cos (x) \cos (y)$ are illustrated in Fig. 2. 

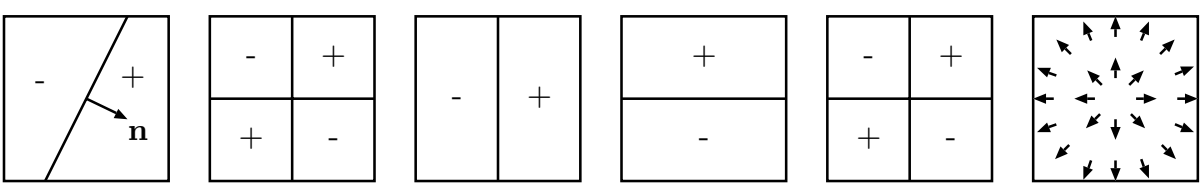

Fig. 1. Symmetries of the odd filters obtained from the considered quadrature approaches. From left to right: the partial Hilbert transform with preference direction $\mathbf{n}$, the total Hilbert transform, the three imaginary components of the quaternionic analytic signal, and the Riesz transform (vector valued)
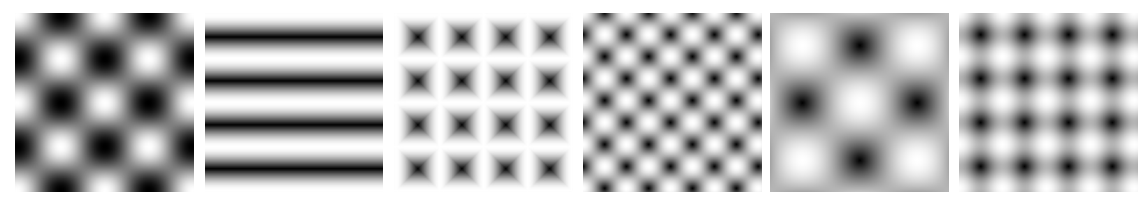

Fig. 2. Amplitudes of the various known approaches to the $2 \mathrm{D}$ analytic signal for a simple 2D signal. From left to right: original signal (black indicates -1 and white indicates 1), the local amplitudes obtained from the partial Hilbert transform with respect to $x$, the orientation adaptive Hilbert transform, the total Hilbert transform, the quaternionic analytic signal, and the monogenic signal. All amplitude images are in a range from zero (black) to one (white). For the quaternionic analytic signal the signal has been rotated by $\pi / 4$

\subsection{Other Related Approaches}

Talking about the analysis of intrinsically $1 \mathrm{D}$ and 2D signals, it is natural to consider also the structure tensor $[12,13]$. The structure tensor can either be computed by a set of classical quadrature filters (see e.g. [2]) or by partial derivatives (see e.g. [14]). In the latter case, the structure tensor can be considered in the context of an approximation of the autocorrelation of the signal [15]. Hence, the two eigenvalues of the structure tensor are related to the principal curvatures of the autocorrelation function, whereas the eigenvectors indicate the corresponding coordinate system. Therefore, the structure tensor can be used to estimate the main orientation of a structure by means of the eigenvector which corresponds to the larger eigenvalue.

The eigenvalues themselves and their relation also provide a measure for the intrinsic dimension of the signal. In [14], the coherence is used for this purpose and it is defined by the square ratio of the difference and the sum of the eigenvalues. However, there exist other definitions for coherence measures in the literature (see e.g. [16]).

According to [2], the structure tensor fulfills the invariance - equivariance requirement with respect to rotations and changes of the signal structure. The estimation of the local orientation is independent of the underlying shape of the signal (e.g. line-like or edge-like) and its energy, whereas the coherence and the norm of the tensor are rotation invariant. Hence, the design principle of the structure tensor is to some extent related to the properties of quadrature 
filters, i.e., local features are mutually independent. The new approach which is presented below extends the invariance - equivariance property to the full superset of features obtained from quadrature filters (i.e., local phase and local amplitude) and from the structure tensor (i.e., local orientation, local coherence, and a local intensity measure).

\section{The New Approach}

As pointed out in the introduction, it is desirable to have an approach which combines the properties of quadrature filters and of the structure tensor in order to obtain a sophisticated $2 \mathrm{D}$ analytic signal. The theory which is developed in the sequel is based on a signal model which is similar to that of the structure tensor.

\subsection{The 2D Signal Model}

Intrinsically $2 \mathrm{D}$ signals have a much greater variety than $1 \mathrm{D}$ signals. Actually, the number of possible 2D signals is infinite times larger than the (already infinite) number of $1 \mathrm{D}$ signals. This increase of possible signal realizations can be considered more formally by means of symmetries. Whereas $1 \mathrm{D}$ signals can be distinguished into locally even and locally odd functions, a 2D signal can contain infinite many even or odd 1D functions with different orientations.

In order to analyze arbitrary 2D signals with respect to all components, infinite many basis filters are necessary. The local signal analysis could be thought of as a spherical Fourier series which is known to have infinite many basis function. This is true unless the signals under consideration are sampled. In the latter case it is not reasonable to apply basis filters with arbitrary high angular frequencies. If the main coefficients of the basis filters are obtained from the samples adjacent to the origin, it is even necessary to restrict the spherical Fourier basis functions to be of order less or equal to three in order to avoid aliasing. Hence, the signal model introduced below is not a heuristic choice but it is the most complete local model which does not suffer from angular aliasing.

As we will show in the subsequent section, the restriction of the basis functions directly correspond to a decomposition of $2 \mathrm{D}$ signals into two perpendicular $^{1}$ intrinsically 1D signals. The Fourier basis functions of order zero to three establish a 4D space of angular functions. Since each of the two involved 1D functions consist of an odd and an even part, two perpendicular 1D functions can be expressed as a $4 \mathrm{D}$ vector. Accordingly, we define the following local $2 \mathrm{D}$ signal model which will be used in the sequel to approximate arbitrary $2 \mathrm{D}$ functions. Let $f_{1}$ and $f_{2}$ be two arbitrary 1D signals and let $\mathbf{n}(\mathbf{x})=(\cos \theta(\mathbf{x}), \sin \theta(\mathbf{x}))^{T}$ be

\footnotetext{
${ }^{1}$ We use the term perpendicular for two $1 \mathrm{D}$ signals with orthogonal orientation vectors. Opposed to that, orthogonality of signals refers to their linear independence in the vector space of functions.
} 
a unit vector with direction $\theta(\mathbf{x})$ where $\mathbf{x}=(x, y)^{T}$ indicates the spatial vector. Then, the $2 \mathrm{D}$ signal $\tilde{f}(\mathbf{x})$ is obtained as

$$
\tilde{f}(\mathbf{x})=f_{1}(\mathbf{x} \cdot \mathbf{n}(\mathbf{x}))+f_{2}\left(\mathbf{x} \cdot \mathbf{n}^{\perp}(\mathbf{x})\right)
$$

where $\mathbf{n}^{\perp}(\mathbf{x})$ is the vector obtained by rotating $\mathbf{n}(\mathbf{x})$ by $\pi / 2$ anticlockwise.

Any 2D signal can be approximated by an appropriate choice of such a 2D signal $\tilde{f}$. Considering the original signal and its approximation in the Fourier domain, the approximation corresponds to a suppression of all frequency components which are not lying either on the main orientation line or on the line perpendicular to the main orientation. However, Fourier theory and a correct estimate of the main orientation ensure that this approximation is $L^{2}$ optimal.

\subsection{The Basis Functions}

For the sake of a more formal investigation, consider the following signal representation. Apart from the ordinary 2D Fourier basis, an arbitrary 2D function can be represented by means of the following set of basis functions:

$$
\begin{aligned}
& b_{1}(\mathbf{x}, \mathbf{u})=\cos (2 \pi x u) \cos (2 \pi y v) \\
& b_{2}(\mathbf{x}, \mathbf{u})=\cos (2 \pi x u) \sin (2 \pi y v) \\
& b_{3}(\mathbf{x}, \mathbf{u})=\sin (2 \pi x u) \cos (2 \pi y v) \\
& b_{4}(\mathbf{x}, \mathbf{u})=\sin (2 \pi x u) \sin (2 \pi y v)
\end{aligned}
$$

see e.g. [8]. Each of these basis functions can be rewritten by means of the trigonometric addition theorems as sums of trigonometric functions:

$$
\begin{aligned}
& b_{1}(\mathbf{x}, \mathbf{u})=(\cos (2 \pi(x u-y v))+\cos (2 \pi(x u+y v))) / 2 \\
& b_{2}(\mathbf{x}, \mathbf{u})=(-\sin (2 \pi(x u-y v))+\sin (2 \pi(x u+y v))) / 2 \\
& b_{3}(\mathbf{x}, \mathbf{u})=(\sin (2 \pi(x u-y v))+\sin (2 \pi(x u+y v))) / 2 \\
& b_{4}(\mathbf{x}, \mathbf{u})=(\cos (2 \pi(x u-y v))-\cos (2 \pi(x u+y v))) / 2 .
\end{aligned}
$$

The basis functions $b_{1}, \ldots, b_{4}$ also establish a basis if the coordinate system is rotated. Moreover, the representation of the rotated basis functions as sums of trigonometric functions is nothing else but a signal representation according to the $2 \mathrm{D}$ model which has been introduced in the previous section since each basis function consists of two perpendicular harmonic oscillations.

The signal which has been used in Fig. 2 is the basis function $b_{1}\left(\mathbf{x},(1,1)^{T}\right)$. Obviously, all known approaches to the $2 \mathrm{D}$ analytic signal fail to decompose the latter function into its amplitude and phase information, except for the quaternionic analytic signal. The latter yields a constant (and therefore correct) amplitude for $b_{1}$ but it fails if the latter is rotated by $\pi / 4$ (see also Fig. 2).

In order to understand what happens if the amplitude is estimated correctly, consider Fig. 3. These illustrations show the basis functions $b_{1}, \ldots, b_{4}$ in the frequency domain with respect to the coordinate system spanned by $\mathbf{n}$ and $\mathbf{n}^{\perp}$. 

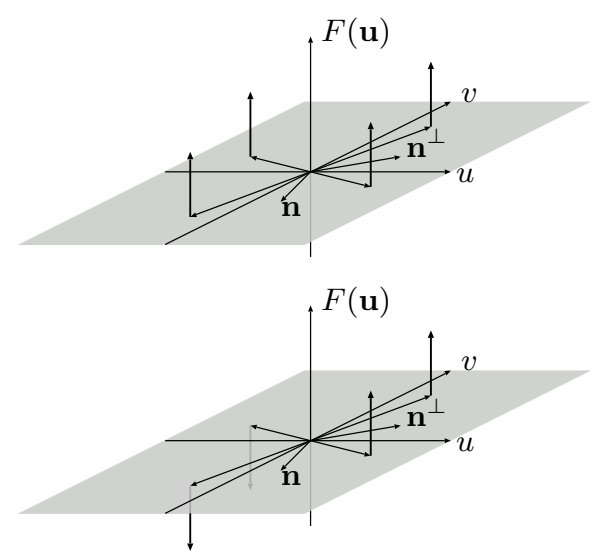
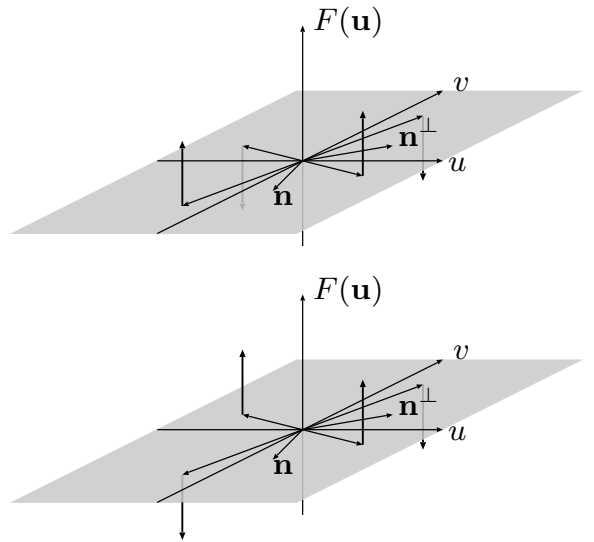

Fig. 3. The basis functions $B_{1}, \ldots, B_{4}$ for $\mathbf{u}=(1,1)^{T}$ with respect to the coordinate system spanned by $\mathbf{n}$ and $\mathbf{n}^{\perp}$. Upper left: $B_{1}$, bottom left: $i B_{2}$, upper right: $i B_{3}$, bottom right: $-B_{4}$

The sum of all four functions yields just one impulse in the quadrant between $\mathbf{n}$ and $\mathbf{n}^{\perp}$. Setting the other three quadrants to zero is similar to the idea of the quaternionic analytic signal with the important difference that the quadrants are not fixed to the coordinate system but they are attached to the vector $\mathbf{n}$.

The principle of quadrature in 1D is to replace pairs of impulses in the frequency domain (i.e., the Fourier transform of an arbitrary harmonic oscillation) with a single impulse. In 2D however, this principle can be extended in several ways. If intrinsically $1 \mathrm{D}$ signals are to be considered, it is sufficient to replace pairs of impulses with a single impulse. However, if intrinsically 2D signals are to be considered, quadruples or $2 n$-tuples have to be replaced with one single impulse. The number of applied basis functions must be identical to the number of impulses which are to be replaced. Hence, intrinsically 2D approaches require at least four basis functions but can potentially be infinitely dimensional. According to the previous discussion about the signal model we retain with quadruples of impulses and hence, with four basis functions.

\subsection{General Formulation}

Up to now it is unclear how to obtain rotation invariant basis functions although the method to be applied has already been mentioned: spherical harmonics. Spherical harmonics allow to design steerable filters [6] which are orientation adaptive and therefore, rotation invariant.

The spherical harmonics of order 1,2, and 3 are illustrated as vector fields in Fig. 4. The vector fields are obtained according to the frequency response

$$
H_{n}(\mathbf{u})=\left(\frac{u+i v}{|\mathbf{u}|}\right)^{n}
$$



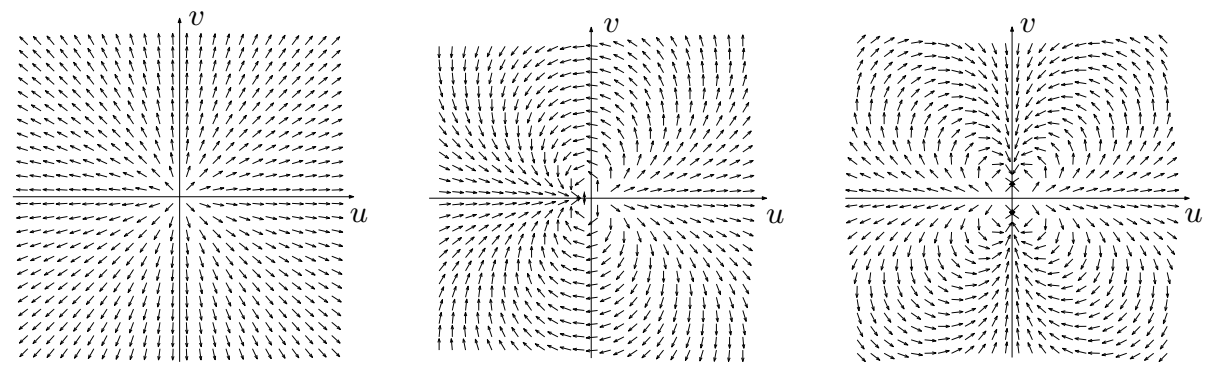

Fig. 4. Spherical harmonics of order 1, 2, and 3 (from left to right) illustrated as vector fields

where $H_{1}(\mathbf{u})$ is basically identical to the Riesz transform. By appropriate steering operations and linear combinations, the vector fields can be used to create the desired symmetry properties sketched in Fig. 3.

The symmetry of $B_{1}$ is trivial and is obtained by a simple allpass frequency response. The symmetry of $B_{4}$ is obtained by projecting the second spherical harmonic onto the double-angle orientation vector (see Fig. 5 , left). If $\theta_{0}$ indicates
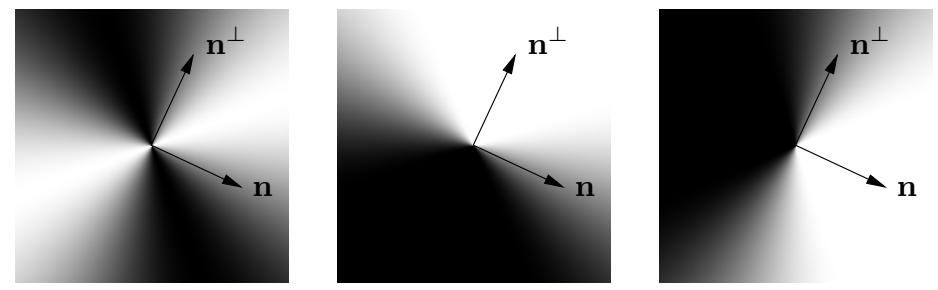

Fig. 5. From left to right: projection of the second order harmonic, linear combination and projection of odd order spherical harmonics to achieve $B_{2}$ symmetry, and linear combination and projection of odd order spherical harmonics to achieve $B_{3}$ symmetry

the main orientation (for the estimation of the main orientation see next section), the $B_{4}$-symmetry is obtained according to

$$
B_{4}(\mathbf{u})=\operatorname{real}\left\{\exp \left(-i 2 \theta_{0}\right) H_{2}(\mathbf{u})\right\}=\cos \left(2\left(\theta-\theta_{0}\right)\right) .
$$

Note that the main orientation of the signal is not identical to the argument of $\mathbf{n}$ but lies on the diagonal of the quadrant between $\mathbf{n}$ and $\mathbf{n}^{\perp}$ (see also Fig. 3).

The two basis functions $B_{1} \equiv 1$ and $B_{4}$ can be combined in order to obtain the angular windowing functions

$$
\begin{aligned}
& W_{1}(\mathbf{u})=1+B_{4}(\mathbf{u})=2 \cos ^{2}\left(\theta-\theta_{0}\right) \\
& W_{2}(\mathbf{u})=1-B_{4}(\mathbf{u})=2 \sin ^{2}\left(\theta-\theta_{0}\right) .
\end{aligned}
$$

The angular shape of these window functions is the same as for the filters which are involved in the calculation of the structure tensor $[2,14]$. 
The remaining two symmetries can be obtained by applying $H_{1}(\mathbf{u})$ (i.e., the Riesz transform) to the two windowing functions and steering them. Since $H_{1}(\mathbf{u})$ is complex valued, the resulting functions are also complex valued:

$$
\begin{aligned}
W_{3}^{\prime}(\mathbf{u}) & =\exp \left(-i \theta_{0}\right) H_{1}(\mathbf{u}) W_{1}(\mathbf{u}) \\
& =\cos \left(\theta-\theta_{0}\right)+\frac{1}{2}\left(\exp \left(i 3\left(\theta-\theta_{0}\right)\right)+\exp \left(i\left(\theta-\theta_{0}\right)\right)\right) \\
W_{4}^{\prime}(\mathbf{u}) & =\exp \left(-i \theta_{0}\right) H_{1}(\mathbf{u}) W_{2}(\mathbf{u}) \\
& =i \sin \left(\theta-\theta_{0}\right)-\frac{1}{2}\left(\exp \left(i 3\left(\theta-\theta_{0}\right)\right)-\exp \left(i\left(\theta-\theta_{0}\right)\right)\right) .
\end{aligned}
$$

Since we need scalar valued functions rather than complex valued ones, the frequency responses $W_{3}^{\prime}$ and $W_{4}^{\prime}$ must be projected without changing the amplitude or losing the antisymmetry. This would be done by considering the (signed) absolute value of the frequency response. However, taking the absolute value is not linear and therefore, this operation cannot be transferred to the spatial domain, which is necessary in order to steer the responses. Fortunately, the signed absolute value can be replaced by taking the real part and the imaginary part without introducing large errors. The relative mean square error is less than $2.3 \%$ and it is concentrated on the diagonals between the main orientation and the line perpendicular to it, which means that it only affects those signals which violate the assumed signal model ${ }^{2}$. Hence, the necessary scalar valued functions are obtained as

$$
\begin{aligned}
& W_{3}(\mathbf{u})=\operatorname{real}\left\{W_{3}^{\prime}\right\}=\frac{3}{2} \cos \left(\theta-\theta_{0}\right)+\frac{1}{2} \cos \left(3\left(\theta-\theta_{0}\right)\right) \\
& W_{4}(\mathbf{u})=\operatorname{imag}\left\{W_{4}^{\prime}\right\}=\frac{3}{2} \sin \left(\theta-\theta_{0}\right)-\frac{1}{2} \sin \left(3\left(\theta-\theta_{0}\right)\right) .
\end{aligned}
$$

Adding and subtracting these two functions yields the remaining two basis functions $B_{2}=W_{3}+W_{4}$ and $B_{3}=W_{3}-W_{4}$. The resulting frequency responses can be found in Fig. 5 (center and right) and realize the symmetries according to Fig. 3.

Although the previous results have been developed and illustrated in the frequency domain, the filters, the steering operations, and the various linear combinations are actually applied in the spatial domain, see Fig. 6.

Finally, all of the previously described filters have an allpass amplitude response. In order to decompose the signal into its distinct frequency components, the filters are combined with radial bandpass filters. The choice of the specific bandpass design is not crucial. However, for the subsequent experiments, we have used the difference of Poisson filters bandpass (see [17]) which is similar to the lognormal bandpass (see e.g. [2]).

\footnotetext{
${ }^{2}$ The linear factors $\frac{3}{2}$ and $\frac{1}{2}$ can be numerically optimized which gives a further improvement of the approximation. Note that for 2D signals according to the assumed model, taking the real part and imaginary part yields exact solutions. The approximation however improves the robustness of the approach.
} 

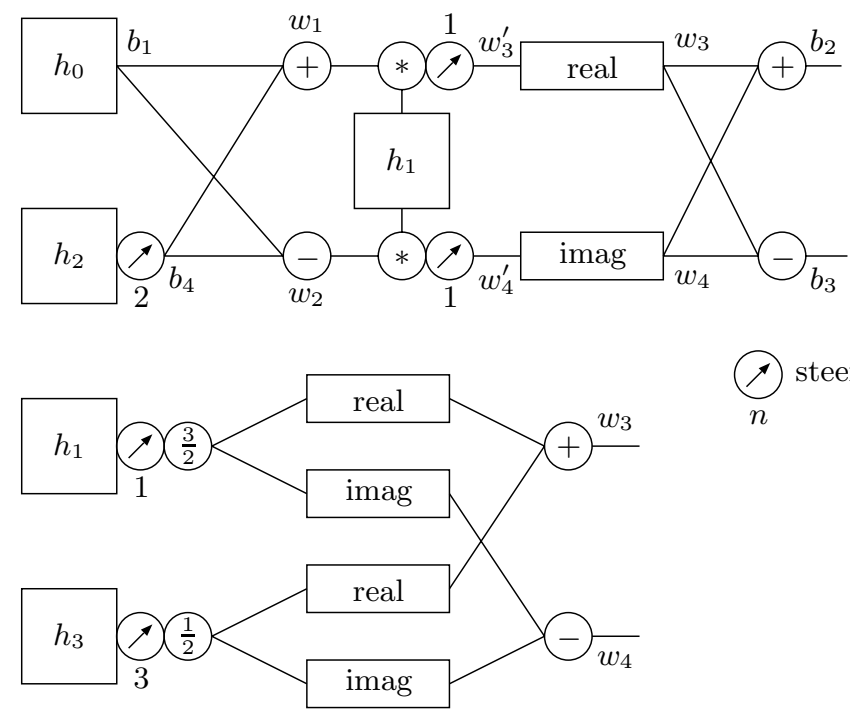

$\bigodot_{n}$ steer with $\exp \left(-i n \theta_{0}\right)$

Fig. 6. Overview of the filter design process. In the upper part of the figure, the filter responses of $b_{2}$ and $b_{3}$ are obtained from those of $b_{1}$ and $b_{4}$ whereas in the bottom part they are directly computed from the spherical harmonics of order one and three

\section{Feature Detection}

From the filter responses produced by $b_{1}, \ldots, b_{4}$ several local properties and features of the signal can be extracted. Although we started the discussion with the basis functions $b_{1}, \ldots, b_{4}$, we will now rather focus upon the responses of $w_{1}, \ldots, w_{4}$. However, since both sets of filters can easily be exchanged there is no fundamental difference.

\subsection{Local Orientation Estimation}

In the previous section, several steering operations have been performed which depend on the local orientation. Although there are several methods for estimating the local orientation which could be applied beforehand, it is more efficient to use the responses of the spherical harmonics to estimate the orientation. However, the spherical harmonics yield phase-dependent estimates of the orientation. Consider e.g. the first order harmonic. For phases close to zero and close to $\pi$ the orientation estimate becomes unreliable (see [18] and Fig. 7). In order to obtain a stable and efficient method for orientation estimation, the impact of the phase to the mean estimation error has to be investigated. In turns out, that the spherical harmonics of odd order and of even order show an opposite behavior (see Fig. 7), which implies that a combination of odd order and even order terms improves the estimate.

It is sufficient to estimate the orientation within $[0, \pi / 2)$ since orientation information is normally within $[0, \pi)$ and due to the supposed signal model, the 

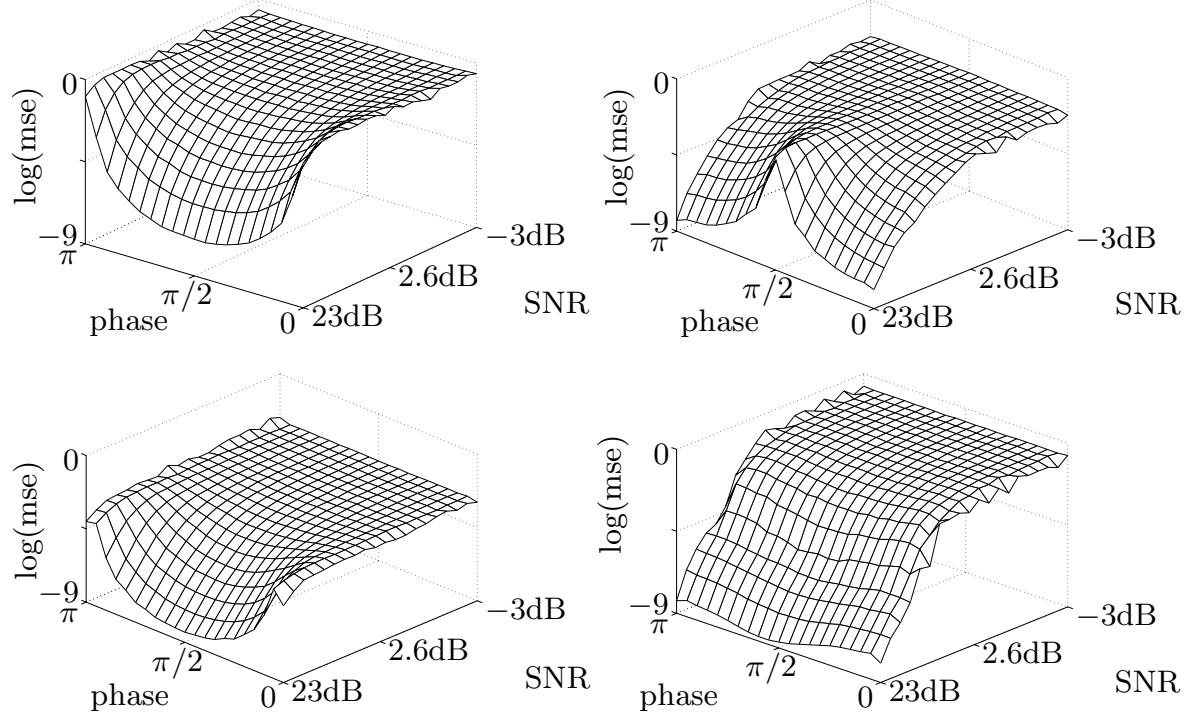

Fig. 7. Phase dependence of the mean square error of some orientation estimate methods. Top left: first order harmonic, top right: second order harmonic, bottom left: third order harmonic, and bottom right: method according to (18). The figures show the logarithm of the mean square error drawn against the local phase and the signal to noise ratio. A value of 0 means an error of $\pi / 2$, which is the maximal possible orientation error. Actually, the orientation errors have been evaluated by taking the second, fourth, and sixth power of a complex exponential, yielding a maximal error of $\pi / 2$, $\pi / 4$, and $\pi / 6$, respectively. Therefore, the error for low SNR seems to decrease with the order of the harmonic which is basically not true. The test signal which has been used for this investigation is a radial modulation, i.e., $\cos \left(\sqrt{x^{2}+y^{2}}\right)$

remaining interval $[\pi / 2, \pi)$ is covered by the perpendicular component. Hence, any product of spherical harmonics which has a maximal order of four can be used to estimate the orientation in the reduced interval. Since the energy of the filter responses is proportional to the square of the sine and of the cosine of the local phase for odd and even order harmonics respectively, a reasonable choice to combine the filter responses for estimating the orientation independent of the phase $^{3}$ is given by

$$
\theta_{\mathrm{e}}=\arg \left(\left(h_{2} * f\right)^{2}+\left(h_{1} * f\right)\left(h_{3} * f\right)\right) / 4 .
$$

The resulting mean square error of this orientation estimation can also be found in Fig. 7. For all subsequent experiments the orientation has been estimated by the previous formula.

\footnotetext{
${ }^{3}$ Actually, the proposed estimate is phase independent for i1D signals. For i2D signals, orientation information can be totally undetermined (e.g. at local extrema) which is also reflected be the proposed method since the magnitude of the complex number in (18) is zero in that case.
} 


\subsection{Local Image Characterization by a Feature Set}

After estimating the local orientation, the responses of the spherical harmonics can be steered such that the responses of $w_{1}, \ldots, w_{4}$ are obtained. Combining the responses of $w_{1}$ and $w_{3}$ according to

$$
f_{\mathrm{A}}(\mathbf{x})=\left(w_{1}(\mathbf{x})+i w_{3}(\mathbf{x})\right) * f(\mathbf{x})
$$

yields a complex signal which picks up signal components close to the estimated orientation. The other two responses, i.e. of $w_{2}$ and $w_{4}$, are combined as

$$
f_{\mathrm{B}}(\mathbf{x})=\left(w_{2}(\mathbf{x})+i w_{4}(\mathbf{x})\right) * f(\mathbf{x}),
$$

which is the signal consisting of components perpendicular to the estimated orientation. Each of the two signals provides a local amplitude and a local phase:

$$
A_{\mathrm{A} / \mathrm{B}}(\mathbf{x})=\left|f_{\mathrm{A} / \mathrm{B}}(\mathbf{x})\right| \quad \text { and } \quad \varphi_{\mathrm{A} / \mathrm{B}}(\mathbf{x})=\arg \left(f_{\mathrm{A} / \mathrm{B}}(\mathbf{x})\right) .
$$

Comparing $A_{\mathrm{A}}$ and $A_{\mathrm{B}}$ yields the dominant partial signal and allows to change the orientation estimate $\theta_{\mathrm{e}}$ into the main orientation: If $A_{\mathrm{A}}(\mathbf{x}) \geq A_{\mathrm{B}}(\mathbf{x})$ the estimated orientation is the main orientation $\theta_{\mathrm{m}}$ and otherwise $\theta_{\mathrm{m}}=\theta_{\mathrm{e}}+\pi / 2$. Furthermore, a measure for the local isotropy is provided by the ratio of the two amplitudes:

$$
c(\mathbf{x})=\min \left\{A_{\mathrm{B}}(\mathbf{x}) / A_{\mathrm{A}}(\mathbf{x}), A_{\mathrm{A}}(\mathbf{x}) / A_{\mathrm{B}}(\mathbf{x})\right\},
$$

so that $c(\mathbf{x})$ is zero if the signal is intrinsically $1 \mathrm{D}$ and it is one if the signal is either intrinsically 2D or constant, i.e., if the energy is distributed isotropically.

The two local amplitudes can be combined to form a total local amplitude measure according to

$$
A_{\mathrm{T}}(\mathbf{x})=A_{\mathrm{A}}(\mathbf{x})+A_{\mathrm{B}}(\mathbf{x}) .
$$

For a synthetic image which is much harder to separate than that in Fig. 2, the following feature images are obtained, see Fig. 8. According to the orientation wrapping of the main orientation at $\pi$ and zero, the phase image shows a phase inversion on that line. The phase of the non-dominant part of the signal is inverted along a vertical line since the orientation-shift of $\pi / 2$ for the perpendicular part moves the orientation wrapping to a vertical line.
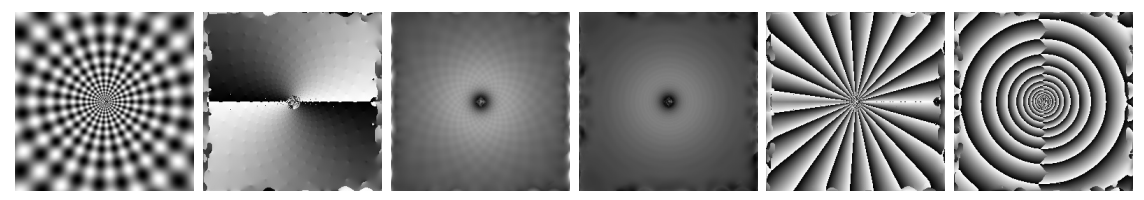

Fig. 8. From left to right: test image consisting of a superposition of an angular and a radial modulation, estimated main orientation, amplitude of the dominant part, amplitude of the non-dominant part, phase of the dominant part, and phase of the non-dominant part. Note the correspondence between orientation wrapping and phase inversion 


\subsection{Detection of Points of Interest}

The energy of the non-dominant structure provides a mean for detecting intrinsically 2D points, e.g., corners, line crossing, or general junctions. This can directly be compared to the Plessey (or Harris-Stephens) detector [19] which is itself closely related to the structure tensor and to coherence measures. In Fig. 9 the results of the new point-of-interest detector are compared to those of the Plessey detector. The new method just detects local maxima of the non-
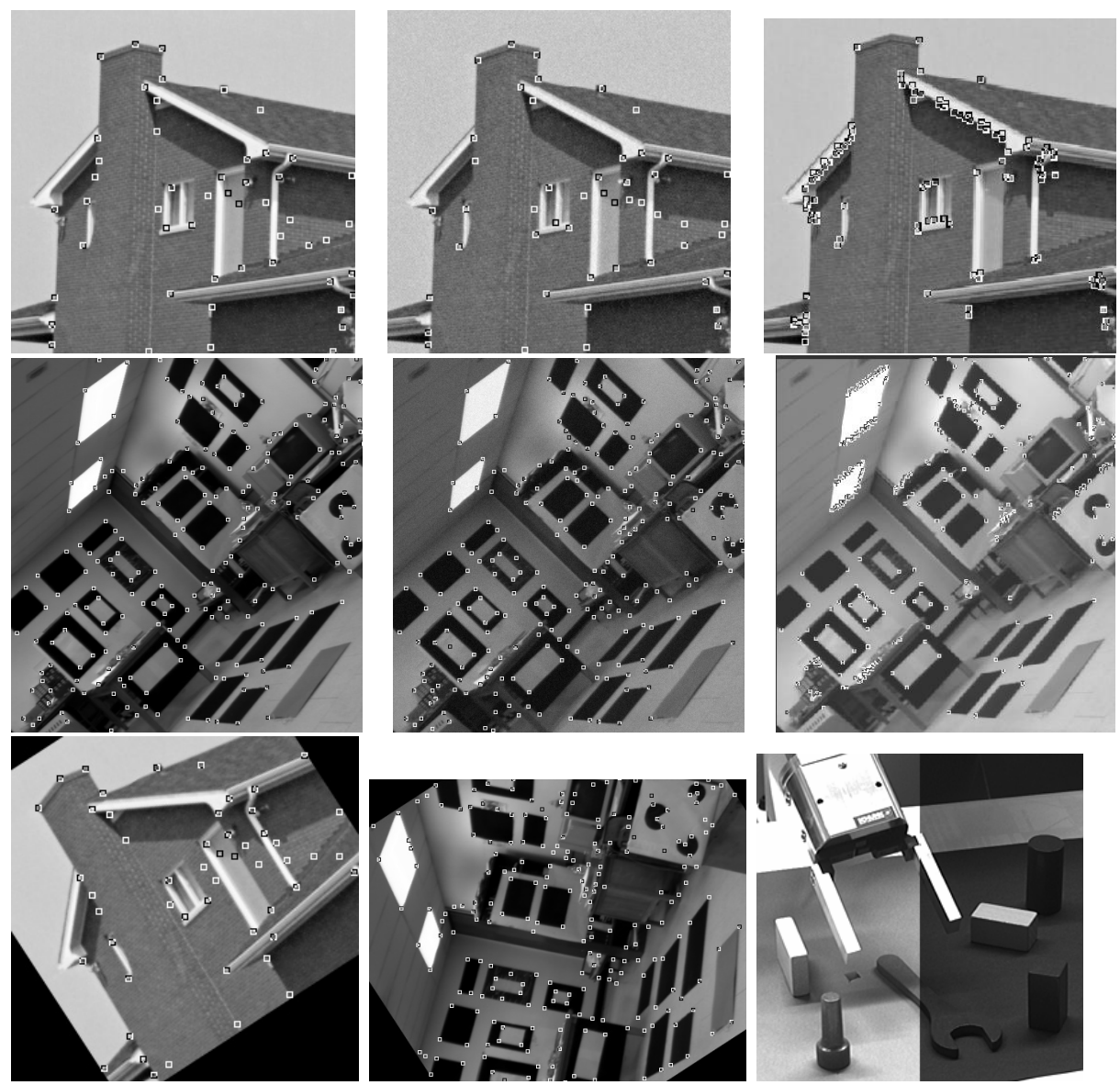

Fig. 9. Upper two rows from left to right: Output of the proposed point-of-interest detector without noise, with noise (white, Gaussian distribution, variance 25), and of the Plessey detector. The original images and the output of the Plessey detector are taken from [20]. Bottom row: detection in rotated images (left and center), the right image shows the reference image (left half) and a test image (right half) for the illumination experiment 
Table 1. Repeatability rates for illumination changes. A detection is repeated if the detected point in the reference image is adjacent to the one in the test image

\begin{tabular}{|l|l|l|l|l|l|l|l|l|l|l|l|l|}
\hline mean $\left(10^{2}\right)$ & 1.56 & 1.20 & 0.92 & 0.71 & 1.75 & 1.44 & 1.09 & 0.79 & 1.28 & 1.02 & 0.81 & 0.60 \\
var $\left(10^{3}\right)$ & 4.80 & 4.06 & 2.91 & 2.08 & 5.71 & 4.69 & 3.19 & 2.03 & 4.74 & 4.19 & 3.08 & 2.05 \\
rep. rate & 1.00 & 0.80 & 0.73 & 0.62 & 0.72 & 0.73 & 0.68 & 0.62 & 0.74 & 0.64 & 0.58 & 0.54 \\
\hline mean $\left(10^{2}\right)$ & 1.50 & 1.15 & 0.85 & 0.65 & 1.28 & 1.02 & 0.75 & 0.58 & 1.69 & 1.33 & 1.02 & 0.78 \\
var $\left(10^{3}\right)$ & 4.72 & 4.15 & 2.83 & 1.97 & 4.80 & 4.32 & 2.91 & 2.06 & 5.30 & 4.19 & 2.96 & 2.06 \\
rep. rate & 0.75 & 0.71 & 0.56 & 0.52 & 0.68 & 0.62 & 0.55 & 0.52 & 0.77 & 0.74 & 0.70 & 0.65 \\
\hline
\end{tabular}

dominant local amplitude in a neighborhood with a radius of seven pixels. Only those maxima are kept which are above a certain threshold, where the latter is given by two times the mean amplitude. The results of the proposed method are better than those of the Plessey detector, with respect to false-positives, falsenegatives, and noise-sensitivity. The outputs of other corner detectors like the SUSAN detector, the Kitchen/Rosenfeld detector, and the CSS approach can be found at [20]. The new approach performs nearly as good as the CSS approach and in contrast to the mentioned methods, the new approach also provides information about the kind of the point-of-interest. Hence, it allows to distinguish between corners, endstoppings, line-crossings, etc..

In two further experiments, the detector has been applied to images with added noise and to rotated images. The detector output is fairly the same as in the original experiment. In order to assess the performance of the new detector in further detail, we made a similar investigation as proposed in [21]. We evaluated the repeatability rate for various kinds of different illuminations of the scene in Fig. 9, bottom right. The images are taken from [22]. The results can be found in Tab. 1 where the change of illumination is represented by the mean and the variance of the test images. The results are close to those in [21].

\section{Conclusion}

We have presented a new approach to 2D quadrature filters which generalizes the idea of invariance - equivariance. Combining the features which are obtained from the classical quadrature filters and the structure tensor, our method allows to estimate five features at a time: local orientation, local isotropy, local amplitude, and two local phases. Due to the invariance - equivariance property, all features are mutually independent of each other.

As an application we have presented a corner detector and compared it to other approaches. Although the local phases at the detected point of interest allow to classify that point, further investigations have to be done in order to obtain a stable algorithm. However, the results from the synthetic image in Fig. 8 show that the presented method yields reliable estimates of the local phases. Furthermore, the proposed algorithm is quite efficient, since it consists of only one real valued and three complex valued convolutions and of three complex multiplications for the steering operations. 


\section{References}

1. Oppenheim, A., Lim, J.: The importance of phase in signals. Proc. of the IEEE 69 (1981) 529-541

2. Granlund, G.H., Knutsson, H.: Signal Processing for Computer Vision. Kluwer Academic Publishers, Dordrecht (1995)

3. Koenderink, J.J.: What is a "feature"? Journal of Intelligent Systems 3 (1993) 49-82

4. Krieger, G., Zetzsche, C.: Nonlinear image operators for the evaluation of local intrinsic dimensionality. IEEE Trans. on Image Processing 5 (1996) 1026-1041

5. Bülow, T., Sommer, G.: The hypercomplex signal - a novel approach to the multidimensional analytic signal. IEEE Trans. on Signal Processing 49 (2001) 2844-2852

6. Freeman, W.T., Adelson, E.H.: The design and use of steerable filters. IEEE Trans. on Pattern Analysis and Machine Intelligence 13 (1991) 891-906

7. Hahn, S.L.: Hilbert Transforms in Signal Processing. Artech House, Boston, London (1996)

8. Bülow, T., Sommer, G.: Algebraically extended representation of multi-dimensional signals. In: Proc. of the 10th Scand. Conf. on Image Analysis. (1997) 559-566

9. Sangwine, S.J.: Fourier-transforms of color images using quaternion or hypercomplex numbers. Electronic Letters 32 (1996) 1979-1980

10. Felsberg, M., Sommer, G.: The monogenic signal. IEEE Trans. on Signal Processing 49 (2001) 3136-3144

11. Stein, E., Weiss, G.: Introduction to Fourier Analysis on Euclidean Spaces. Princeton University Press, New Jersey (1971)

12. Bigün, J., Granlund, G.H.: Optimal orientation detection of linear symmetry. In: Proc. of the IEEE First Intern. Conference on Computer Vision. (1987) 433-438

13. Förstner, W., Gülch, E.: A fast operator for detection and precise location of distinct points, corners and centres of circular features. In: ISPRS Intercommission Workshop, Interlaken. (1987) 149-155

14. Jähne, B.: Digitale Bildverarbeitung. Springer-Verlag, Berlin (1997)

15. Förstner, W.: Statistische Verfahren für die automatische Bildanalyse und ihre Bewertung bei der Objekterkennung und -vermessung. Number 370 in C. Verlag der Bayerischen Akademie der Wissenschaften (1991)

16. Weickert, J.: A review of nonlinear diffusion filtering. In ter Haar Romeny, B., Florack, L., Koenderink, J., Viergever, M., eds.: Scale-Space Theory in Computer Vision. Volume 1252 of LNCS., Springer, Berlin (1997) 260-271

17. Felsberg, M., Sommer, G.: Scale adaptive filtering derived from the Laplace equation. In: 23. DAGM Symposium Mustererkennung, München. Volume 2191 of LNCS., Springer-Verlag, Heidelberg (2001) 124-131

18. Felsberg, M., Sommer, G.: A new extension of linear signal processing for estimating local properties and detecting features. In: 22. DAGM Symposium Mustererkennung, Kiel. Springer-Verlag, Heidelberg (2000) 195-202

19. Harris, C.G., Stephens, M.: A combined corner and edge detector. In: 4th Alvey Vision Conference. (1988) 147-151

20. Mokhtarian, F.: Image corner detection through curvature scale space. http:// www.ee.surrey.ac.uk/Research/VSSP/demos/corners/ (2001) (Accessed 16 Nov 2001).

21. Schmid, C., Mohr, R., C., B.: Evaluation of interest point detectors. International Journal of Computer Vision 37 (2000) 151-172

22. Pauli, J.: Kiel appearance image library. http://www.ks.informatik.uni-kiel. de/ jpa/images.html (1998) (Accessed 22 Feb 2002). 